\title{
Comparison of different protein extraction methods for gel-based proteomic analysis of Ganoderma spp.
}

\begin{abstract}
Ganoderma species are a group of fungi that have the ability to degrade lignin polymers and cause severe diseases such as stem and root rot and can infect economically important plants and perennial crops such as oil palm, especially in tropical countries such as Malaysia. Unfortunately, very little is known about the complex interplay between oil palm and Ganoderma in the pathogenesis of the diseases. Proteomic technologies are simple yet powerful tools in comparing protein profile and have been widely used to study plantï fungus interaction. A critical step to perform a good proteome research is to establish a method that gives the best quality and a wide coverage of total proteins. Despite the availability of various protein extraction protocols from pathogenic fungi in the literature, no single extraction method was found suitable for all types of pathogenic fungi. To develop an optimized protein extraction protocol for 2-DE gel analysis of Ganoderma spp., three previously reported protein extraction protocols were compared: trichloroacetic acid, sucrose and phenol/ammonium acetate in methanol. The third method was found to give the most reproducible gels and highest protein concentration. Using the later method, a total of 10 protein spots ( 5 from each species) were successfully identified. Hence, the results from this study propose phenol/ammonium acetate in methanol as the most effective protein extraction method for 2-DE proteomic studies of Ganoderma spp.
\end{abstract}

Keyword: Basal stem rot; Extraction protocol; Ganoderma boninense; Ganoderma tornatum; Two-dimensional gel electrophoresis 